\title{
A generalization of generalized Banach fixed point theorem in a weak left small self-distance space
}

\author{
F. M. Zeyada, M. R. A. Moubarak, and A. H. \\ Soliman
}




\title{
A GENERALIZATION OF GENERALIZED BANACH FIXED-POINT THEOREM IN A WEAK LEFT SMALL SLF-DISTANCE SPACE
}

\author{
F. M. ZEYADA, M. R. A. MOUBARAK, AND A. H. SOLIMAN \\ Received 8 September, 2004
}

\begin{abstract}
In this paper we generalize the multivalued contraction theorem of S. B. Nadler [8]. As corollaries we obtain fixed point theorems for a multivalued function in complete dislocated metric spaces and complete partial metric space.
\end{abstract}

1991 Mathematics Subject Classification: 54H25, 47H10

Keywords: fixed point, generalized metric spaces

\section{INTRODUCTION}

The generalized Banach fixed point Theorem was introduced in [8]. Hitzler and Seda (2000) introduced the dislocated metrics space in $[3,4]$ as a generalization of metrics where self-distances need not be zero. Also dislocated metrics were studied under the name of metric domains in the context of domain theory in [1]. The notion of dislocated metric is useful in the context of electronic engineering (see [3]). In 1985, S. G. Matthews [6] introduced a generalization of Banach contraction principle. In 2000, Hitzler and Seda [4] introduced an alternative proof of this result in dislocated metric spaces. The plan of this paper is as follows. In Section 2, some preliminaries are presented. In Section 3, we define a weak left small self distance space and give a new topology on a distance space different from the distance topology of P. Waszkiewicz $[9,10]$. In Section 4, we generalize the multivalued contraction theorem by S. B. Nadler [8]. As corollaries of this result we obtain fixed point theorems for a multivalued function in a complete dislocated metric space and a complete partial metric space.

\section{Preliminaries}

In this section we give some preliminaries.

Definition $1([9,10])$. A distance on a set $X$ is a map $d: X \times X \rightarrow[0, \infty)$. A pair $(X, d)$ is called a distance space. 
Let $\varepsilon>0$ and $x \in X$. Then

$$
B_{d}(x, \varepsilon)=\{y \in X \mid d(x, y)<d(x, x)+\varepsilon\}
$$

and $N_{x}=\left\{A \subseteq X \mid \exists \varepsilon>0, B_{d}(x, \varepsilon) \subseteq A\right\}$. The family $\tau_{d}=\{A \subseteq X \mid \forall x \in A, A \in$ $\left.N_{x}\right\}$ is a topology on $X$ and called the distance, the topology $\tau_{d}$ is called the distance topology. If $d(x, y)$ is replaced by $d(y, x)$ it is called the dual distance topology.

We need the following conditions for a distance function:

(d1) $\forall x \in X, d(x, x)=0$,

(d2) $\forall x, y \in X, d(x, y)=d(y, x)=0 \Rightarrow x=y$,

(d3) $\forall x, y \in X, d(x, y)=d(y, x)$,

(d4) $\forall x, y, z \in X, d(x, y) \leq d(x, z)+d(z, y)$,

(d5) $\forall x, y \in X, d(x, x) \leq \min \{d(x, y), d(y, x)\}$,

(d6) $\forall x, y, z \in X, d(x, y) \leq d(x, z)+d(z, y)-d(z, z)$.

If $d$ satisfies conditions (d1)-(d4), then $(X, d)$ is called a metric space. If it satisfies conditions $(\mathrm{d} 2)-(\mathrm{d} 4)$, then $(X, d)$ is called a dislocated metric space (d-metric space for short). If $d$ satisfies conditions (d2), (d3), (d5) and (d6) then $(X, d)$ is called a partial metric space [7].

One can deduce that every partial metric space is a dislocated metric space.

Definition 2. Let $(X, d)$ be a distance space. A multivalued function $f$ on $A \subseteq X$ is a function $f: A \rightarrow 2^{A}-\{\phi\}$.

Theorem 1 ([3,6]). Let $(X, d)$ be a complete d-metric space and let $f: X \rightarrow X$ be a Banach contraction function. Then $f$ has a unique fixed point.

Theorem 2 ([8]). Let $(X, d)$ be a complete metric space and $A \subseteq X$. Suppose that $f$ is a multivalued function on $A, A$ is closed, $f(x)$ is closed for all $x \in A, D$ is the Hausdorff metric, and multivalued $k$-contraction condition

$$
D(f(x), f(y)) \leq k d(x, y)
$$

is satisfied for all $x, y \in A$ and fixed $k \in[0,1)$. Then $f$ has a fixed point, i.e., $\exists x \in A$ such that $x \in f(x)$.

Theorem 3 ([2,5]). Let $(X, d)$ be a metric space. A mapping $T$ from $X$ to itself is said to be asymptotically regular if and only if

$$
\lim _{n \rightarrow \infty} d\left(T^{n}(x), T^{n+1}(x)\right)=0, \forall x \in X .
$$

\section{ON DistANCE SPACES}

Definition 3. Let $(X, d)$ be a distance space and let $A \subseteq X$.

(1) The left $d$-closure of $A$ is denoted and defined by

$$
d_{l}-\operatorname{cl}(A)=\left\{x \in X \mid \exists\left(x_{n}\right) \subset X, \lim _{n \rightarrow \infty} d\left(x_{n}, A\right)=\lim _{n \rightarrow \infty} d\left(x_{n}, x\right)=0\right\}
$$

if $A \neq \phi$, and $d_{l}-\operatorname{cl}(\phi)=\phi$. 
(2) $A$ is called left- $d$-closed $\left(d_{l}\right.$-closed for short) iff $d_{l}-\operatorname{cl}(A) \subseteq A$,

(3) For $a \in X$ and $B \subseteq X$, we put $d(a, B)=\inf _{b \in B} d(a, b)$ and $d(B, a)=$ $\inf _{b \in B} d(b, a)$.

(4) For $A \subseteq X, B \subseteq X$, we put $d(A, B)=\inf \{d(a, b) \mid a \in A, b \in B\}$,

(5) The Hausdorff distance $D$ on $X$ is defined by

$$
D(A, B)=\max \left\{\sup _{a \in A} d(a, B), \sup _{b \in B} d(A, b)\right\},
$$

where $A \subseteq X, B \subseteq X$.

In [8, Theorem 2.2], above the definition of Hausdorff metric coincides with the definition of Hausdorff distance iff $d$ is a metric.

Definition 4. A sequence $\left(x_{n}\right)$ in a distance space $(X, d)$ is said to be left convergent to an $x \in X$ (respectively, asymptotically regular) iff $\lim _{n \rightarrow \infty} d\left(x_{n}, x\right)=0$ (respectively, $\lim _{n \rightarrow \infty} d\left(x_{n}, x_{n+1}\right)=0$ ). If $\lim _{n \rightarrow \infty} d\left(x_{n}, x\right)=0$, then $x$ is called a left limit of $\left(x_{n}\right)$.

If $(X, d)$ is a metric space and $T$ is a mapping from $X$ to itself, then $T$ is asymptotically regular iff $\forall x \in X$, the sequence $T^{n}(x)$ is left asymptotically regular.

It is clear that every left Cauchy sequence (see Definition 4.2 below) is left asymptotically regular. The converse is not true even in metric spaces, for instance let $X=\mathbb{R}$ and $d(x, y)=|x-y| \forall x, y \in X$, then $\left(x_{n}\right)$ in $X$ defined by $x_{n}=\sum_{k=1}^{n} k^{-1}$ is asymptotically regular but not Cauchy.

Definition 5. A distance space is called complete left asymptotically regular distance iff every left asymptotically regular sequence $\left(x_{n}\right)$ in $X$, left converges to some point in $X$.

Theorem 4. Let $(X, d)$ be a distance space. Then

$$
\tau_{d_{l}}=\left\{A \mid A \in 2^{X} \text { and } A^{c} \text { is } d_{l} \text {-closed }\right\}
$$

is a topology on $X$, where $A^{c}$ is the complement of $A$.

Proof. (i) Since $d_{l}-\operatorname{cl}(\phi)=\phi \subseteq \phi$, then $\phi$ is $d_{l}$-closed and so $X \in \tau_{d_{l}}$. Since $d_{l}-\operatorname{cl}(x) \subseteq X$, then $X$ is $d_{l}$-closed and so $\phi \in \tau_{d_{l}}$.

(ii) Let $A, B \in \tau_{d_{l}}, x \in d_{l}-\operatorname{cl}\left(A^{c} \cup B^{c}\right)$ then, there is a sequence $\left(x_{n}\right)$ such that

$$
\lim _{n \rightarrow \infty} d\left(x_{n}, A^{c} \cup B^{c}\right)=\lim _{n \rightarrow \infty} d\left(x_{n}, x\right)=0,
$$

this implies that either

$$
\lim _{n \rightarrow \infty} d\left(x_{n}, A^{c}\right)=\lim _{n \rightarrow \infty} d\left(x_{n}, x\right)=0
$$

or $\lim _{n \rightarrow \infty} d\left(x_{n}, B^{c}\right)=\lim _{n \rightarrow \infty} d\left(x_{n}, x\right)=0 \Rightarrow x \in d_{l}-\operatorname{cl}\left(A^{c}\right)$ or $x \in d_{l}-$ $\operatorname{cl}\left(B^{c}\right) \Rightarrow x \in A^{c} \cup B^{c}=(A \cap B)^{c}$. 
(iii) Let $\left\{A_{j} \mid j \in J\right\} \subseteq \tau_{d_{l}}$ and $x \in d_{l}-\operatorname{cl}\left(\bigcap_{j \in J} A_{j}^{c}\right)$. Then there exists a sequence $\left(x_{n}\right)$ such that $\lim _{n \rightarrow \infty} d\left(x_{n}, \bigcap_{j \in J} A_{j}^{c}\right)=\lim _{n \rightarrow \infty} d\left(x_{n}, x\right)=0$. Therefore, $\lim _{n \rightarrow \infty} d\left(x_{n}, A_{j}^{c}\right)=\lim _{n \rightarrow \infty} d\left(x_{n}, x\right)=0$ for all $j \in J \Rightarrow x \in d_{l}-\operatorname{cl}\left(A_{j}^{c}\right) \forall j \in J$ $\Rightarrow x \in \bigcap_{j \in J} A_{j}^{c}=\left(\bigcup_{j \in J} A_{j}\right)^{c}$.

Definition $6([9,10])$. A distance space $(X, d)$ is called a small self-distance iff $d$ satisfies the condition (d5) above.

Definition 7. A distance space $(X, d)$ is called a weak left small self-distance iff $d$ satisfies the condition $d(x, y)=0 \Rightarrow d(x, x)=0, \forall x, y \in X$.

It is clear that any small self distance space is weak left small self-distance. The following counterexamples illustrate that $\tau_{d_{l}}$ and the distance topology $\tau_{d}$ by P. Wasztiewiecz are different concepts even in a weak left small self distance space.

Counterexample 1. Let $X=\{a, b, c\}$ and $d: X \times X \rightarrow[0, \infty)$ is defined by the relation $d(x, y)=1$ for all $x, y \in X$. It is clear that $(X, d)$ is a weak left small self distance space. Since $\{a\} \notin \tau_{d}$ and $\tau_{d_{l}}=2^{X}$, it follows that $\tau_{d_{l}} \nsubseteq \tau_{d}$.

Counterexample 2. Let $X=\{a, b, c\}$ and $d: X \times X \rightarrow[0, \infty)$ is defined as follows: $d(b, a)=d(b, b)=0, d(a, a)=2^{-1}$ and $d(x, y)=2$ for all $(x, y) \in X \times X-$ $\{(b, a),(b, b),(a, a)\}$. Then $(X, d)$ is a weak left small self distance space. Since $\{a\} \in \tau_{d}$ and $\{a\} \notin \tau_{d_{l}}$, it follows that $\tau_{d} \nsubseteq \tau_{d_{l}}$.

\section{MAIN RESUlTS}

The following counterexample illustrates that there exists a sequence in a weak left small self distance satisfying (d4) which is left convergent but not left asymptotically regular.

Counterexample 3. Let $X=\{a, b, c\}$ and $d$ is a distance function defined by the relations $d(a, b)=d(b, a)=d(c, b)=d(c, a)=d(c, c)=1, d(b, c)=d(a, c)=$ $d(b, b)=d(a, a)=0$. The desired sequence is $\left(x_{n}\right)$ given by the formula

$$
x_{n}= \begin{cases}a & \text { if } n \text { is odd } \\ b & \text { if } n \text { is even }\end{cases}
$$

Definition 8. Let $(X, d)$ be a distance space and $A \subseteq X$. $A$ is called left-asymptotically regular-closed iff if $\left(x_{n}\right)$ is a left asymptotically regular sequence left convergent to $x \in X$, then $x \in A$.

Theorem 5. Let $(X, d)$ be a complete left asymptotically regular weak left small self distance space and $A \subseteq X$. If $f$ is a multivalued function on $A, A$ is leftasymptotically regular-closed, $f(x)$ is left-d-closed $\forall x \in A$ and $f$ satisfies the following condition: $D(f(x), f(y)) \leq k d(x, y) \forall x, y \in A$ for fixed $k \in(0,1)$, then $f$ has a fixed point. 
Proof. (I) Choose a $k_{0} \in(k, 1)$ and an $x_{0} \in A$. If there is not an element $x_{1} \in$ $f\left(x_{0}\right)$ such that $x_{1} \neq x_{0}$, then since $f\left(x_{0}\right) \neq \phi$ we have $x_{0} \in f\left(x_{0}\right)$. Thus $x_{0}$ is a fixed point of $f$. If there exists $x \in f\left(x_{0}\right)$ and $x \neq x_{0}$ such that $d\left(x_{0}, x\right)=0$, then $d\left(x_{0}, f\left(x_{0}\right)\right)=0$. Consider the sequence $\left(x_{n}\right)_{n \in \mathbb{N}}$ where $x_{n}=x_{0} \forall n \in \mathbb{N}$. Then $\lim _{n \rightarrow \infty} d\left(x_{n}, f\left(x_{0}\right)\right)=\lim _{n \rightarrow \infty} d\left(x_{n}, x_{0}\right)=0$, because $(X, d)$ is a weak left small self distance space, i. e., since $d\left(x_{0}, x\right)=0$ we have $d\left(x_{0}, x_{0}\right)=0$. Thus $x_{0} \in d_{l}-\operatorname{cl}\left(f\left(x_{0}\right)\right) \subseteq f\left(x_{0}\right)$. Hence $x_{0}$ is a fixed point. For the other case choose $x_{1} \in f\left(x_{0}\right)$ such that $x_{1} \neq x_{0}$ and $d\left(x_{0}, x_{1}\right)>0$. Now,

$$
d\left(x_{1}, f\left(x_{1}\right)\right) \leq D\left(f\left(x_{0}\right), f\left(x_{1}\right)\right)<k_{0} d\left(x_{0}, x_{1}\right) .
$$

Then there exists $x_{2} \in f\left(x_{1}\right)$ such that $d\left(x_{1}, x_{2}\right)<k_{0} d\left(x_{0}, x_{1}\right)$. By repeating this process one can construct a sequence $\left(x_{n}\right)$ such that $x_{n+1} \in f\left(x_{n}\right), d\left(x_{n}, x_{n+1}\right)<$ $k_{0}^{n} d\left(x_{0}, x_{1}\right)$ for all $n \in \mathbb{N}$. Thus $\lim _{n \rightarrow \infty} d\left(x_{n}, x_{n+1}\right)=0$ and so $\left(x_{n}\right)$ is a left asymptotically regular sequence. Since $(X, d)$ is a complete left asymptotically regular distance space, then exists $x \in X$ such that $\lim _{n \rightarrow \infty} d\left(x_{n}, x\right)=0$. Since $A$ is left-asymptotically regular-closed, then $x \in A$.

(II) Since $d\left(x_{n+1}, f(x)\right) \leq D\left(f\left(x_{n}\right), f(x)\right) \leq k d\left(x_{n}, x\right) \forall n \in \mathbb{N}$, then

$$
\lim _{n \rightarrow \infty} d\left(x_{n+1}, f(x)\right) \leq k \lim _{n \rightarrow \infty} d\left(x_{n}, x\right)=0,
$$

i. e., $\lim _{n \rightarrow \infty} d\left(x_{n+1}, f(x)\right)=0$. Now,

$$
\lim _{n \rightarrow \infty} d\left(x_{n+1}, f(x)\right)=\lim _{n \rightarrow \infty} d\left(x_{n+1}, x\right)=0 \Rightarrow x \in d_{l}-\operatorname{cl}(f(x)) \subseteq f(x)
$$

then $x \in f(x)$.

Proposition 1. Let $(X, d)$ be a distance space and $A \subseteq X$. Let $f$ be a multivalued function on $A$ such that

(1) $D(f(x), f(y))=0 \forall x, y \in A$,

(2) $\exists x \in A$ such that

(a) $x \in f(\ell)$ for some $\ell \in A$,

(b) $d(x, f(x))=0 \Rightarrow x \in f(x)$.

Then $f$ has a fixed point.

Definition 9. Let $(X, d)$ be a distance space. $A$ sequence $\left(x_{n}\right)$ in $X$ is called left Cauchy iff $\lim _{n \rightarrow \infty} d\left(x_{n}, x_{m}\right)=0 \forall n, m \in \mathbb{N}$ such that $m>n$.

Definition 10. A distance space $(X, d)$ is called left complete iff every left Cauchy sequence in $X$, left converges.

Definition 11. Let $(X, d)$ be a distance space and $A \subseteq X$. $A$ is called left-Cauchyclosed iff if ( $\left.x_{n}\right)$ is a left Cauchy sequence left convergent to $x \in X$, then $x \in A$.

One can prove that the sequence $\left(x_{n}\right)$ defined in (I) in the proof of Theorem 5 is left Cauchy if the triangular inequality holds. So one can have the following theorem. 
Theorem 6. Let $(X, d)$ be a left complete left small self distance spacesuch that $d$ satisfying (d4) and let $A \subseteq X$. If $f$ is a multivalued function on $A, A$ is leftCauchy-closed, $f(x)$ is left-d-closed $\forall x \in A$ and $f$ satisfies the following condition: $D(f(x), f(y)) \leq k d(x, y) \forall x, y \in A$ for fixed $k \in(0,1)$, then $f$ has a fixed point.

Remarks. (1) If $d$ satisfies (d3) the term "left" in each concept is omitted.

(2) Let $(X, d)$ be a distance space satisfies (d3). If $A$ is closed; i. e., if every convergent sequence in $A$ converges in $A$; then $A$ is left-asymptotically regular-closed.

(3) If $(X, d)$ satisfies (d3) and (d2), then $(X, d)$ is a weak small self distance.

From Theorem 6 and the remarks above one can have the following corollaries.

Corollary 1. Let $(X, d)$ be a complete d-metric space and $A \subset X$. If $f$ is a multivalued function on Asuch that $\forall x \in A, A$ is closed, $f(x)$ is $d$-closed and $f$ satisfies the condition mentioned in Theorem 6, then $f$ has a fixed point.

Corollary 2. If $(X, d)$ is a complete partial metric space and $A \subseteq X$. If $f$ is a multivalued function on Asuch that $\forall x \in A, A$ is closed, $f(x)$ is $d$-closed and $f$ satisfies the condition mentioned in Theorem 5, then $f$ has a fixed point.

The following corollary illustrates that Theorem 6 and Proposition 1 generalize the generalized $k$-contraction fixed point Theorem by S. B. Nadler.

Corollary 3 ([8]). Let $(X, d)$ be a complete metric space and $A \subseteq X$. Suppose that $f: A \rightarrow 2^{A}-\{\phi\}$ is a multivalued map such that $A$ is closed, $f(x)$ is closed for all $x \in A$, and multivalued $k$-contraction condition

$$
D(f(x), f(y)) \leq k d(x, y)
$$

is satisfied for all $x, y \in A$ and fixed $k \in[0,1)$. Then $f$ has a fixed point.

The proof follows from the above notes and the following fact: In metric spaces, the topology $\tau_{d_{l}}$ coincides with the usual metric topology.

\section{Acknowledgement}

The authors wish to express their appreciation to the anonymous referees and to Professor Ioan A. Rus from the Babeş-Bolyai University of Cluj-Napoca (Romania) for careful reading and many helpful comments that improved the original manuscript.

\section{REFERENCES}

[1] S. Abramsky and A. Jung, "Domain theory," in Handbook of logic in computer science. New York: Oxford Univ. Press, 1994, vol. 3, pp. 1-168.

[2] F. E. Browder and W. V. Petryshyn, "The solution by iteration of linear functional equations in Banach spaces,” Bull. Amer. Math. Soc., vol. 72, pp. 566-570, 1966. 
[3] P. Hitzler, "Generalized metrics topology in logic programming semantics," Ph.D. dissertation, National University of Ireland, University College Cork, 2001.

[4] P. Hitzler and A. K. Seda, "Dislocated topologies." J. Electr. Eng., vol. 51, no. 12/s, pp. 3-7, 2000.

[5] W. A. Kirk, "Nonexpansive mappings and asymptotic regularity," Nonlinear Anal., vol. 40, no. 1-8, pp. 323-332, 2000.

[6] S. G. Matthews, "Metric domains for completeness," Ph.D. dissertation, Department of Computer Science, University of Warwick, United Kingdom, 1986.

[7] S. G. Matthews, "Partial metric topology," in Proceedings of the 8th Summer Conference on Topology and its Applications, vol. 728, 1992, pp. 183-197.

[8] S. B. Nadler, Jr., "Multi-valued contraction mappings," Pacific J. Math., vol. 30, pp. 475-488, 1969.

[9] P. Waszkiewicz, "Quantitative continuous domains," Ph.D. dissertation, University of Birmingham, 2002.

[10] P. Waszkiewicz, "Quantitative continuous domains," Appl. Categ. Structures, vol. 11, no. 1, pp. 41-67, 2003.

Authors' addresses

\section{F. M. Zeyada}

Department of Mathematics, Faculty of Science, Al-Azhar University, Assiut, Egypt

E-mail address: mhsm20060yahoo.com

M. R. A. Moubarak

Department of Mathematics, Faculty of Science, Minia University, Minia, Egypt

E-mail address: mramobarak@yahoo.com

\section{A. H. Soliman}

Department of Mathematics, Faculty of Science, Al-Azhar University, Assiut, Egypt

E-mail address: a_h_gsoliman@yahoo.com 